\title{
Defining giant mandibular ameloblastomas - Is a separate clinical sub-entity warranted?
}

\author{
Aditya V. Kanoi, Tibar Banerjee, Narayanamurthy Sundaramurthy, Arindam Sarkar, Pooja Kanoi", \\ Sushovan Saha \\ Department of Plastic and Reconstructive Surgery, Institute of Postgraduate Medical Education and Research, Kolkata, \\ West Bengal, 'Department of Prosthodontics and Crown and Bridge, Manipal College of Dental Sciences, Mangaluru, \\ Karnataka, India
}

Address for correspondence: Dr. Narayanamurthy Sundaramurthy, Department of Plastic and Reconstructive Surgery, Institute of Postgraduate Medical Education and Research, Kolkata, West Bengal, India. E-mail: sskm.plasticsurgery@gmail.com

\section{ABSTRACT}

Context: The term giant mandibular ameloblastoma (GMAs) while being in popular usage in the medical literature remains largely equivocal. Although a few authors have in the past attempted to ascribe definite criteria to this entity, these are by and large arbitrary and without any benefit in decision-making or contributing to its management. Aims: The aim of this study is to propose a set of objective criteria for GMAs that can be clinically correlated and thereby aid in the management of this entity. Patients and Methods: Of a total of 16 patients with ameloblastoma of the mandible presenting at our institute from August 2012 to September 2016, 11 patients were identified as having GMAs as per the criteria proposed. Results: The defects in the mandible following segmental resection ranged from 7 to $11.5 \mathrm{~cm}$ in length (mean: $9.3 \mathrm{~cm}$ ). No clinical or radiological evidence of tumour recurrence was found during a mean follow-up period of 10.7 months (range: 2-28 months). Conclusions: Defining GMA based on objective inclusion and exclusion criteria allows segregation of these lesions, thereby helping to remove ambiguity, simplify decision-making and facilitate communication among treating reconstructive surgeons. Inclusion criteria include: (i) The segmental bone defect following resection with a minimum $1 \mathrm{~cm}$ margin of healthy bone should exceed $6 \mathrm{~cm}$ (ii) The segmental bone defect should involve the central mandibular segment.

\section{KEY WORDS}

Ameloblastoma; fibula free flap; jaw neoplasms; mandibular reconstruction; non-vascularised bone graft; segmental mandibulectomy

\begin{tabular}{|l|l|}
\hline \multicolumn{2}{|c|}{ Access this article online } \\
\hline Quick Response Code: & Website: \\
\hline & www.ijps.org \\
\cline { 2 - 2 } & Dol: \\
\hline
\end{tabular}

This is an open access journal, and articles are distributed under the terms of the Creative Commons Attribution-NonCommercial-ShareAlike 4.0 License, which allows others to remix, tweak, and build upon the work non-commercially, as long as appropriate credit is given and the new creations are licensed under the identical terms.

For reprints contact: reprints@medknow.com

How to cite this article: Kanoi AV, Banerjee T, Sundaramurthy $N$, Sarkar A, Kanoi P, Saha S. Defining giant mandibular ameloblastomas - is a separate clinical sub-entity warranted? Indian J Plast Surg 2018;51:208-15. 


\section{INTRODUCTION}

meloblastomas (also known as Adamantinoma; Eve's disease) are uncommon tumours of the jaw constituting $1 \%-3 \%$ of all jaw tumours. ${ }^{[1]}$ While the term 'Giant ameloblastoma of the mandible' has been used quite liberally in the literature, a unanimous definition of the word 'Giant' remains elusive. A literature review performed using the keywords 'giant mandibular ameloblastoma' and 'giant ameloblastoma of the mandible' revealed that the available information on giant mandibular ameloblastomas (GMA) is largely anecdotal and sparse, majority of which is limited to case reports ${ }^{[2-4 \mid}$ typically describing unusually large mandibular growths that are truly 'giant', even to the untrained eye. The authors feel that the term 'giant' can be better utilised to describe those mandibular ameloblastomas demonstrating certain characteristics that warrant special considerations from a clinical perspective rather than its current usage in portraying peculiarly large, 'reportable' cases of these tumours. Doing so will allow for a rational way to approach and manage this entity which until now has been sidelined as a medical aberration.

Based on our experience with mandibular ameloblastomas and a review of the established literature on the subject, we recommend clinically pertinent objective criteria for the classification of mandibular ameloblastomas that is simple enough for use in everyday clinical practice, promotes better doctor communication and most importantly, has a direct influence in decision-making and management. Furthermore, the treatment and outcome of GMA presenting at our institute and as identified by the new set of criteria have been evaluated, in terms of aesthetic and functional results, patient satisfaction and long-term recurrence.

\section{PATIENTS AND METHODS}

From a treatment standpoint, the following sets of inclusion and exclusion criteria were proposed for a GMA.

\section{Inclusion criteria}

i. The segmental bone defect following resection with a minimum $1 \mathrm{~cm}$ margin of healthy bone should exceed $6 \mathrm{~cm}$

ii. The segmental bone defect should involve the central mandibular segment.

\section{Exclusion criteria}

1. Tumours that are more than $5 \mathrm{~cm}$ in its longest dimension but with a narrow base (requiring either a marginal mandibulectomy or a segmental mandibulectomy creating a horizontal defect of $<6 \mathrm{~cm}$ )

2. Exclusive lateral defects, irrespective of the size of the defect.

Reconstruction of large defects in the lateral aspect of the mandible has provided comparable results with vascularised bone grafts, non-vascularised bone grafts (NVBGs) or with only soft-tissue flaps..$^{[5-10]}$ Hence, vascularised bone grafts in such situations are not proven to be significantly better than other modalities.

We report a retrospective analysis of 16 patients of mandibular ameloblastomas presenting at our institute from August 2012 to September 2016, of which eleven were categorised as GMA consistent with the described criteria [Table 1]. Accordingly, these patients received radical treatment in the form of segmental resection and primary reconstruction with vascularised bone flaps (VBF), NVBGs with local flaps and reconstruction plate with condylar prosthesis.

Five of these patients were excluded based on our exclusion criteria. In one instance, although the gross tumour (expanded along the alveolus with a narrow base) measured $>5 \mathrm{~cm}$ in greatest dimension, the post-resection mandibular defect that needed to be reconstructed was $<6 \mathrm{~cm}$. This patient, however, wanted an autologous bony reconstruction and therefore, received a free fibula flap for reconstruction. In the remaining four instances, the defects either did not involve the central segment at all or did not extend significantly across the midline. These patients were reconstructed variously with free fibula flaps $(n=2)$, NVBG $(n=1)$ and reconstruction plate with attached metallic condylar prosthesis $(n=1)$ as studies showed that non-microsurgical methods were as good as vascularised bone transfers for lateral segment defects. ${ }^{[5-10]}$

Pre-operatively, the patients were evaluated clinically and diagnosis established with biopsy. Imaging studies including panoramic X-ray and computed tomography (CT) facial skeleton with three-dimensional (3D) reconstruction were utilised to facilitate surgical planning.

Segmental mandibular defects were classified according to the 'HCL' method described by Jewer Indian Journal of Plastic Surgery Volume 51 Issue 2 May-August 2018 
Table 1: Clinical summary of all patients treated for giant mandibular ameloblastomas

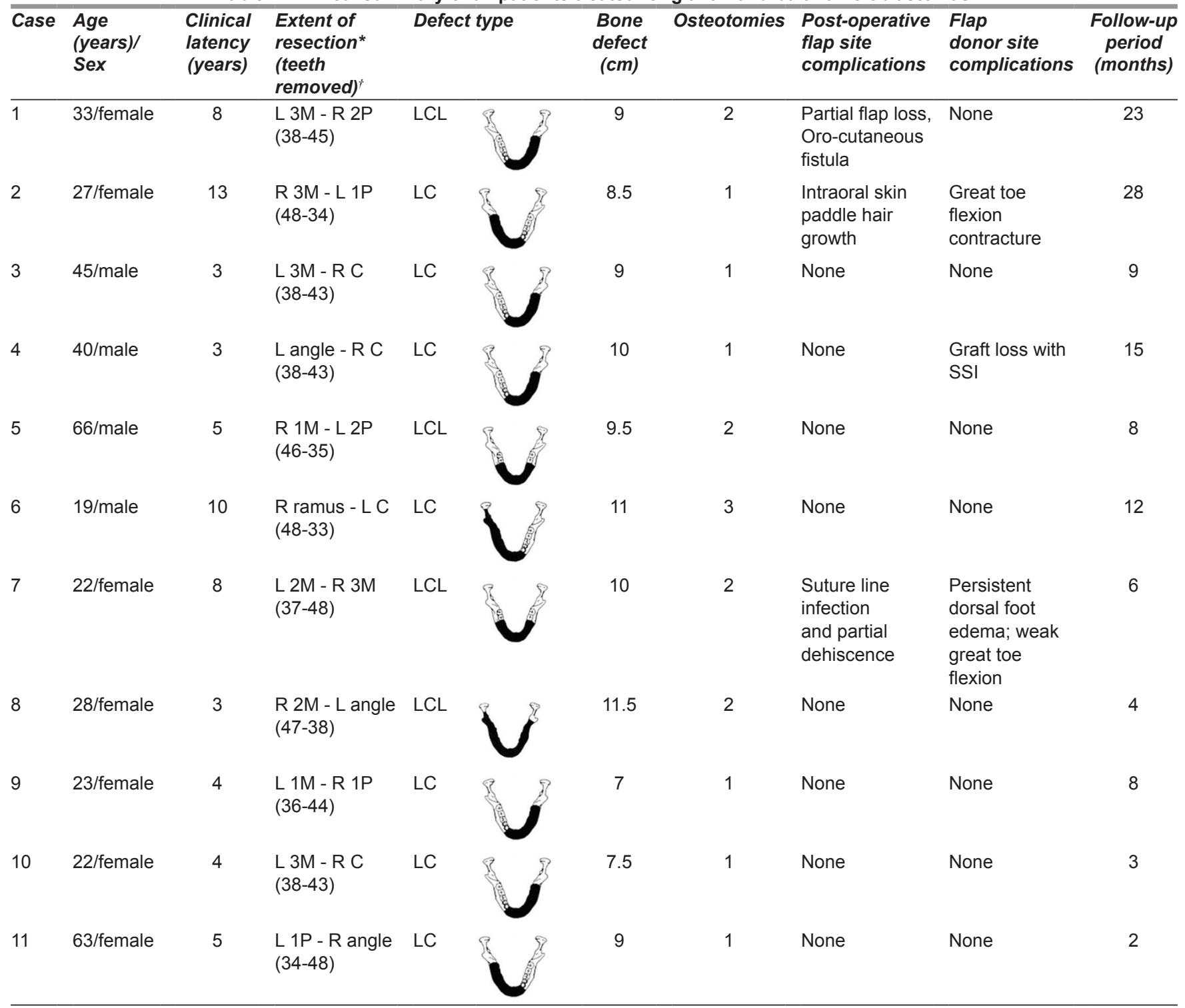

*M: Molar; P: Premolar, C: Canine, ${ }^{\dagger}$ As per the ISO dental notation system. ISO: International Standards Organization, L: Left, R: Right

and later by Boyd et al. ${ }^{[11]}$ Post-operative results were evaluated by the primary author based on two sets of questionnaires, one related to appearance and the other function specific (including speech, chewing and swallowing and drooling of saliva). The approach followed for the management of cases of mandibular ameloblastomas presenting at our institute is depicted in Figure 1.

\section{RESULTS}

Of a total of 16 patients with ameloblastoma of the mandible presenting at our institute during the study period, 11 patients were identified as having GMA as per the criteria proposed [Table 1].
The defects in the mandible following segmental resection ranged from $7 \mathrm{~cm}$ to $11.5 \mathrm{~cm}$ in length (mean: $9.3 \mathrm{~cm}$ ). The left side fibula was consistently used for the transfer for the ease of harvest and patient preference.

All flaps were viable apart from one (90.90\%) (Patient No. 1) who had partial flap necrosis of her skin paddle measuring $8 \mathrm{~cm} \times 5 \mathrm{~cm}$. All resected specimens were subjected to a histopathological examination which showed complete removal of the tumours. Except for Patient No. 1, whose discharge was delayed because of partial flap loss and subsequent development of a small oro-cutaneous fistula that healed on conservative treatment with regular dressings and oral hygiene and Patient No. 4, who developed a surgical site infection 


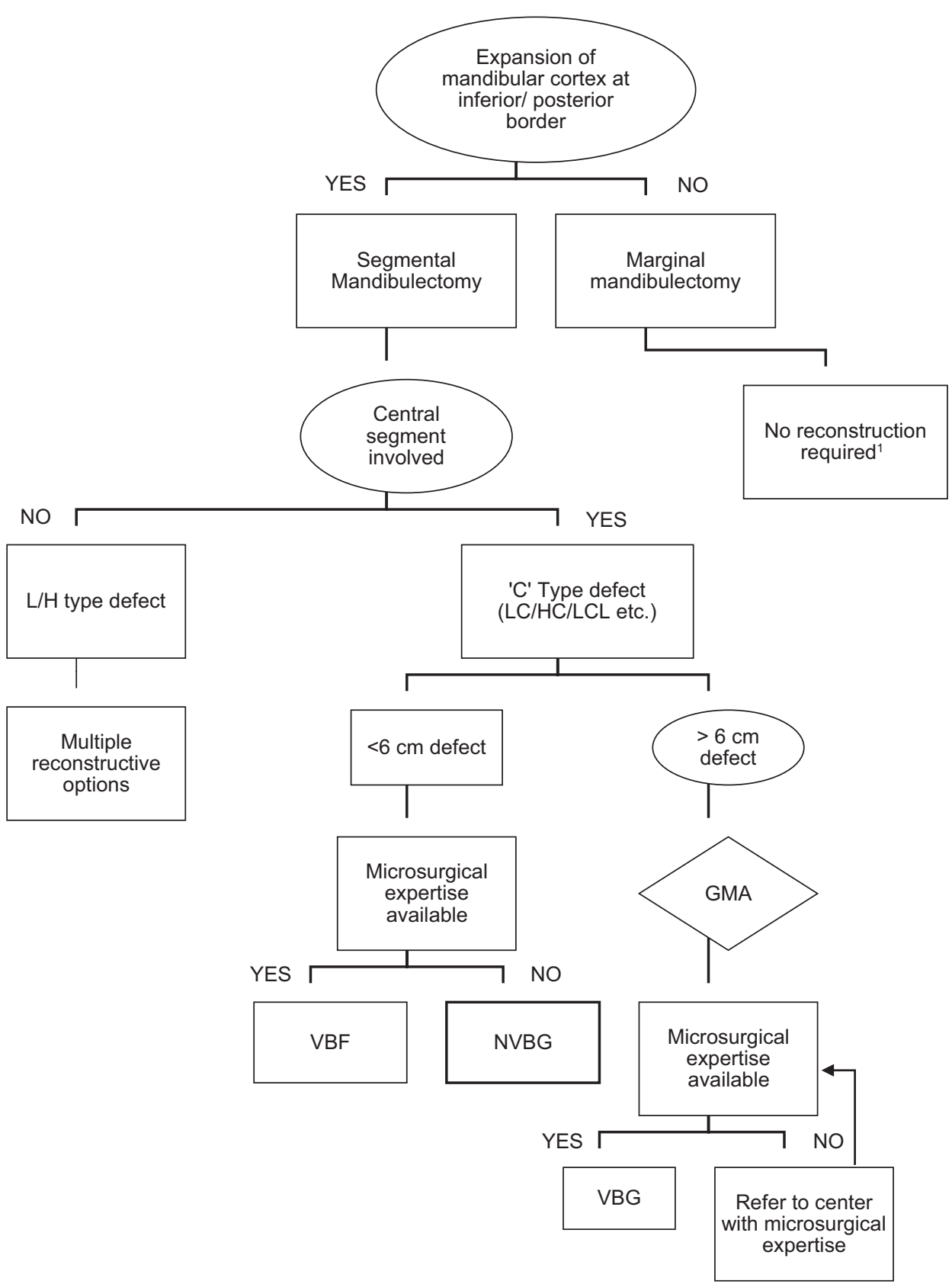

Figure 1: Algorithm depicting step-wise approach to mandibular ameloblastomas. ${ }^{1} \mathrm{~A}$ long, thin rim of residual mandible that is prone to fracture may be supported by the judicious use of reconstruction plates. NVBG: Non-vascularised bone graft, VBF: Vascularised bone flap, GMA: Giant mandibular ameloblastomas

of the flap donor site with subsequent graft loss, hospital stay was $<2$ weeks in the rest of the patients. Complications did not require a return to theatre and were treated conservatively. They did not affect the final result regarding cosmesis and function. At 2-3 months of follow-up, all were tolerating a normal diet and were able to speak coherently and had good oral competence. Although great toe flexion was weak in Patient No. 7 with Patient No. 2 even developing a flexion contracture of her great toe, it did not hamper ambulation. No clinical or radiological evidence of tumour recurrence was found during a mean follow-up period of 10.7 months (range: 2-28 months).

Because of its subjective nature, aesthetic results were assessed by questionnaire-based serial evaluations. Three patients were more than satisfied (score 8-10), six patients were satisfied (score 4-7) and two were not satisfied (score 1-3) with their cosmetic outcomes. The questionnaire was elementary, largely focussing on the patient's ability to integrate socially. Unfortunately, although patients were aggressively counselled Indian Journal of Plastic Surgery Volume 51 Issue 2 May-August 2018 
post-operatively, none of our patients received dental rehabilitation in the form of dental implants.

\section{Patients}

Patient 1-A33-year-old female presented with expansion of the lower jaw for 8 years. The swelling was slow growing, painless and hard in consistency, measuring $12 \mathrm{~cm} \times 9 \mathrm{~cm}$ in greatest dimensions. A CT of the facial skeleton with 3D reconstruction showed a multiloculated lesion with mixed radiolucent and radiopaque areas. Cortical thinning of the inferior mandibular border along with expansion of the alveolus were noted on clinical and radiological examination. A segmental mandibulectomy performed via a submandibular approach with $1 \mathrm{~cm}$ margins extending from $\mathrm{L} 3^{\text {rd }}$ molar to $\mathrm{R} 2^{\text {nd }}$ premolar resulted in a $9 \mathrm{~cm}$ 'LCL' bone defect [Figure 2]. Resection of the overlying mucosa lead to an intraoral lining defect as well. Reconstruction was achieved with a free fibula osteoseptocutaneous flap (FFOSC flap). The flap skin paddle measuring $8 \mathrm{~cm} \times 5 \mathrm{~cm}$ was used to restore the intraoral lining.

Patient 6 - A 19-year-old male presented with a swelling arising from the lower jaw which was slow growing, painless and long-standing ( $>10$ years), measuring $13 \mathrm{~cm} \times 10 \mathrm{~cm}$. The swelling was associated with malocclusion and exfoliation of teeth. An orthopantomogram indicated a bony lesion affecting the $\mathrm{R}$ mandible with mixed radiolucent and opaque

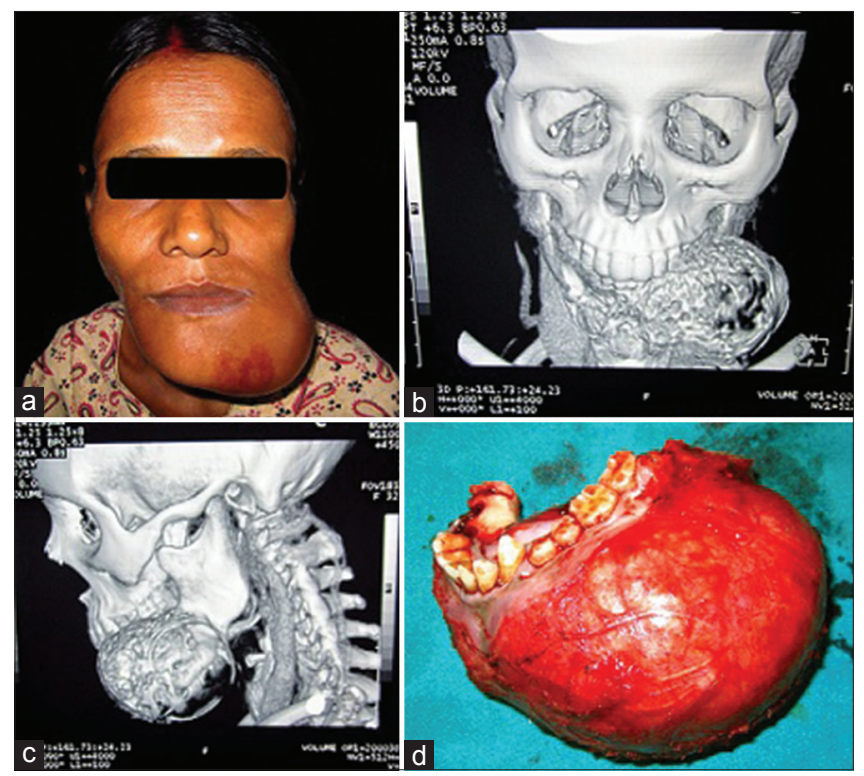

Figure 2: (a). Case 1- a 33-year-old female with $10 \mathrm{~cm} \times 15 \mathrm{~cm}$ mandibular swelling. (b and c) Facial computed tomography showing the multiloculated lesion, anterior and lateral views. (d) Specimen following segmental mandibular resection from the left third molar to right second premolar areas along with alveolar bone destruction. A segmental mandibulectomy was performed through a submandibular approach in combination with a lip-split incision. The resultant $11 \mathrm{~cm}$ 'LC' defect, extending from $\mathrm{R}$ angle to $\mathrm{L}$ canine was reconstructed with a FFOSC flap [Figure 3]. At 6 months of follow-up, he was tolerating a normal diet and could speak coherently.

\section{DISCUSSION}

Although Chaine et al. ${ }^{[12]}$ attempted to define GMA, they did not elucidate the reasons for their criteria. Their criterion of 'large size ( $>5 \mathrm{~cm}$ in length)' is suboptimal because an ameloblastoma often substantially expands and balloons out the mandibular cortex while remaining narrow at its base. Consequently, rather than the size of the ameloblastoma, which may be misleading, the length of the actual defect following its removal with a healthy margin is what is clinically relevant. Furthermore, terms such as 'considerable jaw expansion' seemed arbitrary and lacking clinical significance.

Treatment of ameloblastomas with simple curettage and enucleation is associated with an unacceptably high rate of recurrence. ${ }^{[13]}$ Currently, it is agreed that a radical resection is associated with the lowest recurrence rates on long-term follow-up. ${ }^{[14]}$ Therefore, mandibular resection with a $1-2 \mathrm{~cm}$ margin of healthy bone $\mathrm{e}^{[15,16]}$ is the recommended strategy. This radical resection can be in the form of a segmental or a marginal mandibulectomy.

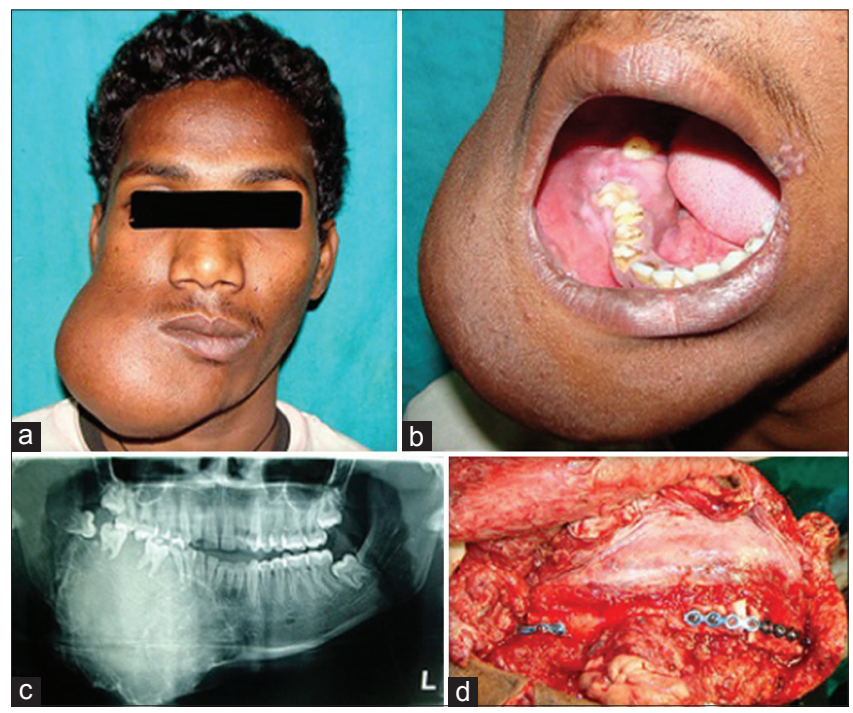

Figure 3: (a). Case 6-slow growing mandibular tumour in a 19-year-old male. (b) Alveolar expansion with malocclusion. (c) Orthopantograph showing exfoliation of teeth. (d) Intra-operative photograph displaying the reconstructed mandible with fibula osteocutaneous flap 
Performing a marginal resection with preservation of the inferior or posterior mandible border, whenever possible, simplifies the reconstruction, especially with regard to the central mandibular defect. ${ }^{[16]}$ A segmental resection should be favoured when the tumour expands the inferior or posterior border or when provisions for close follow-up are lacking. ${ }^{[15]}$

In an article by Pogrel et al..$^{[7]}$ the authors state that NVBGs $>6-9 \mathrm{~cm}$ in length are associated with a graft failure rate of $20 \%$ or more. Similarly, Foster et al.${ }^{[6]}$ showed that the percentage of patients achieving bony union following free fibular flap was almost double (95\% vs. $44 \%)$ compared to NVBGs when performed for mandible defects $6-10 \mathrm{~cm}$ in length. Bearing in mind that free fibula flap failure rates, in general, are $<5 \%-10 \%,{ }^{[17]}$ the use of NVBGs to reconstruct mandibular defects $>6-9 \mathrm{~cm}$ would not be prudent.

Unlike periosteum covering the mandible elsewhere, the alveolar mucoperiosteum does not form an effective barrier to the spread of ameloblastoma. This is evidenced by a lack of capsule formation in this region along with direct contact of the tumour with mucosa ('collision phenomenon') and finally, an infiltrative pattern of growth vis-a-vis the smooth contours seen in the rest of the mandible. ${ }^{[18]} \mathrm{As}$ a consequence, invasion and expansion of the alveolus by the tumour would necessitate inclusion of the overlying oral mucosa in the resection.

Based on a review of cases of mandibular ameloblastomas treated at our institution, a step-wise approach for the management of this entity is illustrated in Figure 1. The decision-making process in the treatment of mandibular ameloblastomas begins with an evaluation of inferior or posterior mandibular border expansion. In its absence, a marginal mandibulectomy can be safely carried out as part of radical treatment while significantly reducing operative complexity, costs and patient morbidity. ${ }^{[15,16]}$ The tumours those fit into the proposed criteria as listed in the ovals implies a GMA and warrants microsurgical expertise for adequate reconstruction.

Tumours that demonstrated alveolar bone invasion and expansion, as determined by clinical and radiological examination, were subjected to excision of the overlying alveolar mucosa followed by reconstruction with the skin paddle of the FFOSC flap. For lateral defects being reconstructed with NVBG or bridging plates, the use of local soft-tissue flaps such as the submental and the facial artery myomucosal flaps can provide alveolar lining in such cases.

Because of the numerous studies in existence, that show that non-microsurgical reconstructive options such as bridging plates and NVBG are adequate modalities for reconstructing lateral mandibular defects,,${ }^{[5-10]}$ we did not feel the need to compare results of VBF reconstruction with other forms of reconstruction and would leave it to the discretion of the treating surgeon to choose the appropriate treatment modality for such defects.

In the light of the present evidence, instead of simply using arbitrary criteria such as size $>5 \mathrm{~cm}$ for defining GMA, we would instead recommend categorising as GMAs those tumours that will need segmental resection leaving a bone defect of $>6 \mathrm{~cm}$ and involving the central segment, thereby necessitating reconstruction with a VBF.

The authors, therefore recommend, that while non-GMA tumours may be treated with a variety of reconstructive approaches including free fibula flaps (based on the surgeon's preference), GMAtumours must be reconstructed with free fibula flaps for optimum outcomes.

Although patients presented with a variety of complaints including but not limited to pain, skin ulceration and dental problems such as malocclusion and loosening of teeth, the primary reason for presentation was a change in appearance. Moreover, all patients retained sufficient oral function which posed a challenge during the reconstructive effort to ensure oral function was not significantly altered. In contrast, based on the authors' experience, oral cancer patients requiring mandibulectomy and mandibular reconstruction (T4b) tend to have more functional problems such as pain, trismus, difficulties in speech and swallowing.

Even though not performed by us, consideration can be given to a double barrel fibula flap ${ }^{[19]}$ to allow easier placement of osseointegrated dental implants. Osseointegrated dental implants will be required for optimal dental rehabilitation. ${ }^{[20]}$ Alternatively, in areas with financial and infrastructure constraints, a more practical and inexpensive option would be the use of removable partial dentures or similar prostheses. ${ }^{[21]}$ Following central mandibular segment resection, the genioglossus (prevents tongue fallback) and digastric (jaw depressor) are inadvertently detached. These 
detached muscles were sutured to empty miniplate holes or around the screws which are then tightened after coiling the suture around them to avoid tongue fall back and lower face and lip sagging ${ }^{[22]}$ in the early post-operative period.

While alternative options for vascularised osteocutaneous flap transfers do exist including circumference iliac osteocutaneous flap and osteocutaneous radial forearm free flap, the free fibula flap was preferred since it offers clear-cut advantages in mandibular reconstruction. ${ }^{[23]}$ Similar to Yadav et al. ${ }^{[24]}$ we felt that choosing one leg over the other as a donor site for flap harvest did not influence the final result. Hence the left leg was consistently used for ease of harvest, intra-operative instrumentation and personnel set-up as well as patient preference.

In most of the patients, because of the large tumour size, often extending to the opposite side, standard techniques for achieving an aesthetically contoured neo-mandible such as pre-plating techniques and CT tracings were difficult to employ. In addition, intermaxillary fixation was not possible in the majority owing to extensive resection of the tooth-bearing mandibular segment. A preformed bent wire template was used to best match the defect. However, the use of dedicated DICOM image processing software such as OsiriX ${ }^{\circledR}$ (Pixmeo; Bernex, Geneva) available for Mac OS (Apple Inc., California) may be of benefit in assisting with pre-operative planning for mandibular reconstructions. ${ }^{[25]}$

\section{CONCLUSIONS}

The clinical presentation, investigation protocols, treatment and follow-up considerations are significantly different for GMA as compared to non-GMA tumours. We believe that infusing the term 'GMA' with the new set of criteria will allow logical segregation of these lesions from non-GMA lesions, thereby facilitating better communication and decision-making among treating reconstructive surgeons. Chiefly, recognising a mandibular ameloblastoma as a GMA should precipitate an early referral to a centre with microvascular expertise thereby expediting the treatment process.

\section{Declaration of patient consent}

The authors certify that they have obtained all appropriate patient consent forms. In the form the patient(s) has/have given his/her/their consent for his/her/their images and other clinical information to be reported in the journal.
The patients understand that their names and initials will not be published and due efforts will be made to conceal their identity, but anonymity cannot be guaranteed.

\section{Acknowledgement}

The authors would like to thank Dr Nicolas Leymarie, MD, MSc, Plastic and Reconstructive Surgery Unit, Gustave Roussy hospital, Villejuif, France, for his assistance in reviewing and editing the manuscript

\section{Financial support and sponsorship}

Nil.

\section{Conflicts of interest}

There are no conflicts of interest.

\section{REFERENCES}

1. Crawley WA, Levin LS. Treatment of the ameloblastoma: A controversy. Cancer 1978;42:357-63.

2. Hughes CA, Wilson WR, Olding M. Giant ameloblastoma: Report of an extreme case and a description of its treatment. Ear Nose Throat J 1999;78:568, 570-2, 574.

3. Dunn JL, Olan WJ, Bank WO, Narang AK, Schwartz AM. Giant ameloblastoma: Radiologic diagnosis and treatment. Radiographics 1997;17:531-6.

4. Kalavrezos N, Baldwin DJ, Walker DM. Giant neglected ameloblastoma: Single stage treatment and clinicopathological review. Br J Oral Maxillofac Surg 2008;46:591-3.

5. van Gemert J, Holtslag I, van der Bilt A, Merkx M, Koole R, Van Cann E, et al. Health-related quality of life after segmental resection of the lateral mandible: Free fibula flap versus plate reconstruction. J Craniomaxillofac Surg 2015;43:658-62.

6. Foster RD, Anthony JP, Sharma A, Pogrel MA. Vascularized bone flaps versus nonvascularized bone grafts for mandibular reconstruction: An outcome analysis of primary bony union and endosseous implant success. Head Neck 1999;21:66-71.

7. Pogrel MA, Podlesh S, Anthony JP, Alexander J. A comparison of vascularized and nonvascularized bone grafts for reconstruction of mandibular continuity defects. J Oral Maxillofac Surg 1997;55:1200-6.

8. Shnayder Y, Lin D, Desai SC, Nussenbaum B, Sand JP, Wax MK, et al. Reconstruction of the lateral mandibular defect: A review and treatment algorithm. JAMA Facial Plast Surg 2015;17:367-73.

9. Boyd JB, Mulholland RS, Davidson J, Gullane PJ, Rotstein LE, Brown $\mathrm{DH}$, et al. The free flap and plate in oromandibular reconstruction: Long-term review and indications. Plast Reconstr Surg 1995;95:1018-28.

10. Head C, Alam D, Sercarz JA, Lee JT, Rawnsley JD, Berke GS, et al. Microvascular flap reconstruction of the mandible: A comparison of bone grafts and bridging plates for restoration of mandibular continuity. Otolaryngol Head Neck Surg 2003;129:48-54.

11. Boyd JB, Gullane PJ, Rotstein LE, Brown DH, Irish JC. Classification of mandibular defects. Plast Reconstr Surg 1993;92:1266-75.

12. Chaine $A$, Pitak-Arnnop $P$, Dhanuthai $K$, Ruhin-Poncet $B$, Bertrand JC, Bertolus C, et al. A treatment algorithm for managing giant mandibular ameloblastoma: 5-year experiences in a Paris university hospital. Eur J Surg Oncol 2009;35:999-1005. 
13. Ghandhi D, Ayoub AF, Pogrel MA, MacDonald G, BrocklebankLM, Moos KF, et al. Ameloblastoma: A surgeon's dilemma. J Oral Maxillofac Surg 2006;64:1010-4.

14. Pogrel MA, Montes DM. Is there a role for enucleation in the management of ameloblastoma? Int J Oral Maxillofac Surg 2009;38:807-12.

15. Feinberg SE, Steinberg B. Surgical management of ameloblastoma. Current status of the literature. Oral Surg Oral Med Oral Pathol Oral Radiol Endod 1996;81:383-8.

16. Maclntosh RB. Aggressive surgical management of ameloblastoma. Oral Maxillofac Surg Clin North Am 1991;3:73-97.

17. Yim KK, Wei FC. Fibula osteoseptocutaneous flap for mandible reconstruction. Microsurgery 1994;15:245-9.

18. Müller $\mathrm{H}$, Slootweg PJ. The growth characteristics of multilocular ameloblastomas. A histological investigation with some inferences with regard to operative procedures. J Maxillofac Surg 1985;13:224-30.

19. Bähr W, Stoll P, Wächter R. Use of the "double barrel" free vascularized fibula in mandibular reconstruction. J Oral Maxillofac Surg 1998;56:38-44.
20. lizuka T, Häfliger J, Seto I, Rahal A, Mericske-Stern R, Smolka K et al. Oral rehabilitation after mandibular reconstruction using an osteocutaneous fibula free flap with endosseous implants. Factors affecting the functional outcome in patients with oral cancer. Clin Oral Implants Res 2005;16:69-79.

21. Carr AB, Bram DT. Partially edentulous epidemiology, physiology and terminology. In: McCracken DJ, editor. Removable Partial Prosthodontics. 12 $2^{\text {th }}$ ed. Missouri: Elsevier Publications; 2011. p. 2-7.

22. al Qattan MM, Boyd JB. Complications in head and neck microsurgery. Microsurgery 1993;14:187-95.

23. Hidalgo DA. Fibula free flap: A new method of mandible reconstruction. Plast Reconstr Surg 1989;84:71-9.

24. Yadav PS, Ahmad QG, Shankhdhar VK, Nambi GI. There is no donor side specificity of fibula free flap for complex oromandibular reconstruction. Indian J Plast Surg 2010;43:177-80.

25. Albert S, Cristofari JP, Cox A, Bensimon JL, Guedon C, Barry B, et al. Mandibular reconstruction with fibula free flap. Experience of virtual reconstruction using osirix $®$, a free and open source software for medical imagery. Ann Chir Plast Esthet 2011;56:494-503. 